\title{
Evaluating the In vivo Efficacy of Copper- Chitosan Nanocomposition for Treating Vascular Wilt Disease in Date Palm
}

\author{
Elwy, A. Mohamed ${ }^{* 1}$; Mohamed, H. Gaber²; Sherif, F. Elsharabasy ${ }^{1}$ \\ ${ }^{1}$ Central Laboratory for Date Palm Research \& Development, Agricultural Research Center, Giza, Egypt. \\ ${ }^{2}$ Department of Biophysics, Faculty of Science, Cairo University, Giza, Egypt.
}

\begin{abstract}
Date palm, Phoenix dactylifera, as one of the most important fruit crops in Egypt and many other countries, can be affected by many fungal diseases, among which the vascular wilt disease, caused by the fungal pathogen Fusarium oxysporum, is considered the most deteriorating one. This study aims at evaluating the efficiency of Copper-Chitosan Nanopcomposition for treating the vascular wilt disease in date palm. The study relies mainly on beleaguering the disease via the doublerole functionality of copper-chitosan nanocomposition, i.e. its potential antifungal effect on the fungal pathogen, besides its capability to enhance the immune responses of the infected plant. In this regard, chitosan nanoparticles were prepared according to the ionic gelation method, whereas copper nanoparticles were prepared according to the chemical reduction method. Physicochemical characterization of both chitosan and copper nanoparticles was performed using dynamic light scattering (DLS), transmission electron microscopy (TEM), fourier transform infrared spectroscopy (FTIR) and $x$-ray diffraction (XRD). Copper-chitosan nanocomposition could significantly reduce the vascular wilt disease severity; this means that the nanocomposition can be used in the future for developing new nanofungicides to control such pathogens.
\end{abstract}

Keywords-Copper nanoparticles, Chitosan nanoparticles, Date palm, Fusarium oxysporum, Vascular wilt.

\section{INTRODUCTION}

Date palm, Phoenix dactylifera, is considered one of the most important fruit crops in many arid regions including the Middle East; This is due to its versatility, as it has a variety of uses includes eating fresh fruit and benefiting from its high nutritional value and its richness of carotenoids, citric acid, folic acid, and provitamins; this in addition to the antiviral, antibacterial, antifungal, antiulcer, antitumor and immunomodulatory properties of phenolic compounds detected in dates. Moreover, this crop has a great potential as a source of renewable energy by producing biofuel due to the high carbohydrates content in the fruits. Also, seeds are used in animal feeding, cosmetics, source of oxalic acid and charcoal, besides using them as a paste to relieve ague (Al-Shahib and Marshall, 2003).

The worldwide production of dates reached $\mathbf{7 6 0 0 3 1 5}$ tons annually. In this context, Egypt is considered the largest producer of dates all over the world with annual production of $\mathbf{1 4 6 5 0 3 0}$ tons (FAO, 2014). This massive production of dates makes date palm a promising potential source of national income in Egypt and other large producers. But, this important crop, like all other crops, is threatened with many fungal and bacterial phytopathogens.

Generally speaking, more than $70 \%$ of the crop diseases result from fungal pathogens (Agrios, G.N., 2005), this indicates to the importance of controlling such virulent phytopathogens. In this regard, surveys showed that among these fungal pathogens, the fungal pathogen Fusarium oxysporum, which causes vascular wilt disease (also known as fusarium wilt disease), is considered the most common and most virulent one (Flood, 2006).

In this context, there are many traditional chemicals that are used to control such pathogenic fungi. But many phytopathogens have exhibited resistance against many traditional chemicals that are used to control them (Hide et.al, 1992; M. J. Hajipour et al., 2012; A. J. Friedman, 2013; Patel et.al., 2014). Thus, there is a dire need to develop newer and more effective controlling agents. In this regard, nanotechnology may provide more efficient alternatives for the current fungicides.

Among different types of nanomaterials, metal nanoparticles have gained a considerable attention and have a wide range of applications due to their unique catalytic, electric, magnetic and structural properties (M. Sahu and P. Biswas, 2011; H. Tian et al., 2014 and H. M. Yadav et al., 2014). In this regard, copper nanoparticles (CuNPs) are considered a very important type of transition metals, which have many potential applications including but not limited to catalysis of some dyes such as rose Bengal dye (S. T. H. Sherazi et al., 2013), printing in electronics (S. Magdassi et al., 2010) 
and its antimicrobial effect (A. D. Karthik and K. Geetha, 2013; Prachi. K. et al., 2013; R. BetancourtGalindo et al., 2014 and A. M. Muthukrishnan et al., 2015).

On the other hand, chitosan has gained much attention in many different applications, including but not limited to pharmaceutical, medical, agricultural, nutritional and industrial applications, this is due to its superior characteristics such as non-toxicity, biodegradability and biocompatibility (Harish P. and Tharanathan, 2007). Also, chitosan nanoparticles (CsNPs) have a potential ability to enhance the immune responses of plants (Chandra, S. et al., 2015), this makes CsNPs a potential competitor in formulating the protective compositions directed toward enhancing the plant immunity.

In this work, the In vivo efficacy of copper-chitosan nanocomposition $(\mathrm{CuCs})$ will be evaluated to treat the vascular wilt disease in date palm. The idea relies upon exploiting the dual functionality of this nanocomposition, i.e. its ability to induce and augment the innate immune responses in the plant and its potential antifungal activity against the fungal pathogen itself. This can hinder the fungal growth inside the vascular system of the infected plant and hence beleaguering the vascular wilt disease.

\section{MATERIALS AND METHODS}

All chemicals used were analytical grade of purity, and were used as received without any further purification.

\subsection{Preparation of CsNPs and CuNPs}

\subsubsection{Preparation of CsNPs}

CsNPs were synthesized in accordance with the ionic gelation method (Qi, L. et al., 2004) with a simple modification, in which:

- $2 \mathrm{~g}$ of chitosan powder (Degree of Deactylation $\geq$ 90\%; water content $\leq 8.0 \%$; Carl Roth, Germany) were dissolved into 1 liter of $1 \%$ acetic acid solution.

- $0.5 \mathrm{~g}$ of sodium tripolyphosphate (TPP) (Sigma Aldrich) was dissolved into 1 liter of distilled water.

- TPP solution was added dropwise under stirring to the chitosan solution in 1:10 volumetric ratio.

- Temperature was kept at $25{ }^{\circ} \mathrm{C}$ and $\mathrm{pH}$ was adjusted at 4 .

\subsubsection{Preparation of CuNPs}

CuNPs were prepared according to the chemical reduction method (Mustafa B. and Ilkay S., 2010), in which Lascorbic acid (0.11M) (Future Modern Co., Egypt) was added into aqueous solution of CTAB (0.09M) (SigmaAldrich, Egypt). $\mathrm{NaOH}$ solution was used to adjust $\mathrm{pH}$ at 6.8; temperature was kept at $85^{\circ} \mathrm{C}$. During stirring of the previous mixture of $\mathrm{CTAB}$ and L-ascorbic acid, Copper sulfate pentahydrate $(0.03 \mathrm{M})$ (Elnasr Pharmacuticals Co., Egypt) solution was introduced. The reaction was continued until reddish brown color was developed. Then, CuNPs were collected by centrifugation for further characterization and application.

\subsection{Characterization of CsNPs and CuNPs}

2.2.1. Fourier Transform Infrared Spectroscopy (FTIR) of CsNPs

Fourier Transform Infrared Spectroscopy (FTIR) was performed to confirm the successful ionic gelation between chitosan and TPP. In this step, solutions of chitosan and CsNPs were firstly lyophilized, using the lyophilizer (EDWARDS Freeze Dryer), then FTIR analysis was performed on the lyophilized chitosan and CsNPs using FTIR spectrophotometer (Model Jasco 4100, Japan; $400-4000 \mathrm{~cm}^{-1}$ ).

\subsubsection{UV-Vis spectroscopy of CuNPs}

The characteristic surface plasmon resonance of CuNPs was detected using Helios Gamma Spectrophotometer.

\subsubsection{X-Ray Diffraction (XRD) of CuNPs}

The characteristic X-Ray Diffractogram of CuNPs was recorded using Philips PW1840 X-Ray Diffractometer, USA $(\lambda=1.54056 \AA ; 40 \mathrm{kV} ; 25 \mathrm{~mA})$. The range of recording was from $5^{\circ}$ to $70^{\circ}$, with a rate of $2^{\circ}$ /minute.

\subsubsection{Determining Particle Size Distribution of CsNPs and CuNPs}

Dynamic light scattering (DLS) (Zetasizer nano series (Nano ZS), Malvern, UK) was used to measure the particles size of the synthesized CsNPs and CuNPs.

\subsubsection{Transmission Electron Microscopy (TEM) of CsNPs and CuNPs}

Transmission Electron Microscopy (Tecnai G20, Super twin, double tilt, FEI, Netherland) was used to figure out the shape of the synthesized CsNPs and CuNPs.

\subsection{The Fungus, Fusarium oxysporum}

Fusarium oxysporum (FO1 isolate), which was isolated from infected date palm, was obtained from Department of Date Palm Pathology, Central Laboratory for Date Palm Research \& Development, Agricultural Research Center, Giza, Egypt.

\subsection{Preparing copper-chitosan nanocomposition (CuCs)}

$\mathrm{CuCs}$ was prepared in four gradient concentrations $(0.5$, 1.0, 1.5 and $2.0 \mathrm{~g} / \mathrm{l}$ ) through the admixture of CuNPs and CsNPs with the respective concentration from both components. 


\subsection{Evaluating the Antifungal Activity of CuCs}

Spores' inoculum of the fungus was prepared; then the microscopic enumeration technique was used to adjust the fungal inoculum size at $1.0 \times 10^{6} \mathrm{spores} / \mathrm{ml}$. After that, $100 \mathrm{ul}$ of the inoculum suspension were used to inoculate each of potato dextrose broth (control) and potato dextrose broth containing different concentrations of the nanocomposition. Both the control and treatments were kept at $30{ }^{\circ} \mathrm{C}$ for 2 days. Then, the optical densities (OD) of the cultures were detected using Uv-Vis Spectrophotometer (ORION AQUAMATE 8000) at 530 nm (Eva Petrikkou et al., 2001). Percentage of inhibition at each concentration was calculated according to the following formula (Ling Yien Ing et al., 2012).

\section{Inhibition rate $=\left(1-O D_{\text {treatment }} / O D_{\text {control }}\right) \times 100$}

\subsection{Evaluating the Immune responses of Date Palm Seedlings caused by $\mathrm{CuCs}$}

Four groups of date palm seedlings, Sewi cultivar, (10 months) were used. Each group consists of 20 healthy seedlings. Each group was irrigated with $50 \mathrm{ml}$ of $\mathrm{CuCs}$ with the respective concentration $(0.5,1.0,1.5$ or $2.0 \mathrm{~g} / \mathrm{l})$ per seedling. The fifth group of seedlings was used as a control, in which seedlings were irrigated with $50 \mathrm{ml}$ water per seedling. Leaves from each seedling were dried, then the total phenolics and enzymes were extracted (kâhkônen et al.,1999).

- The level of total phenolics in the extracts was quantified by the modified Folin - Ciocateu method (Singelton and Rossi, I965). Gallic acid standard (5 $\mathrm{g} \%$ ) was used, and the total phenolic content was expressed as milligram Gallic Acid per gram dry weight of the original sample (mg $\mathrm{GA} / \mathrm{g} \mathrm{dw}$ ).

- Phenoloxidase activity was determined according to (Ishaaya, I971). The phenol oxidase activity was determined as optical density $\left(\mathrm{OD}_{405}\right)$ units $\times 10^{3}$ at an absorbance of $405 \mathrm{~nm}$.

- Peroxidase activity was determined according to (Vetter $\boldsymbol{e t}$ al., I958). The enzyme activity was expressed as the change in absorbance at $430 \mathrm{~nm}$ $\left(\Delta \mathrm{OD}_{430}\right) /$ minute/g fresh weight.

2.7. Evaluation of the In vivo Efficiency of $\mathrm{CuCs}$ in Treating Fusarium Wilted-Date Palm Seedlings In Comparison With Rizolex ${ }^{\mathrm{TM}}$

\subsubsection{Initiation of Fusarium Wilt Disease}

Firstly, the fungal culture was raised on Richard's liquid medium and incubated at $26^{\circ} \mathrm{C}$ for 2 weeks. Thus, the fungal mat is formed (Riker and Riker, 1936). After that, the fungal inoculums were prepared through mixing of $10 \mathrm{~g}$ of the mycelial mat with $100 \mathrm{~mL}$ of distilled water in a blender (S. Ansari et al., 2012). Each seedling was inoculated through adding $50 \mathrm{~mL}$ of the fungal inoculum in its root zone.

\subsubsection{In vivo treatment}

In this experiment, three groups of the inoculated date palm seedlings, Sewi cultivar, (10 months) were used. Each group consists of 20 inoculated seedlings. 10 days after the fungal inoculation, the treatment began as follow:

The first group of inoculated seedlings was used as a treatment, in which $50 \mathrm{ml}$ of $\mathrm{CuCs}$ with different concentrations was uniformly applied to root zone of each inoculated seedling.

The second group of inoculated seedlings was used as a positive control. In which each inoculated seedling was treated with $50 \mathrm{ml}$ of $3 \mathrm{~g} / \mathrm{l}$ Rizolex ${ }^{\mathrm{TM}}$ fungicide.

The third group of the inoculated seedlings was used as a negative control, in which $50 \mathrm{ml}$ of water was added to the root zone of each seedling.

\subsubsection{Assessment Disease Severity}

Disease progression was observed through two successive weeks. Symptoms of Fusarium wilt disease on leaves (wilting and yellowing) of the date palm seedlings were used to measure the disease severity. In this regard, a standard rating (Campbell and Madden, 1990) was used to assess the disease severity for each group. Disease severity (DS) was calculated (Chester et al., 1959; Wheeler, 1969) as follow:

Disease Severity (DS) $=(($ sum of all individual disease rating) / (total number of leaf assessed $\mathrm{x}$ maximum rating) $) \times 100$.

\subsection{Statistical analysis}

SPSS 22 software was used at $P \leq 0.05$ to distinguish between DS, antifungal efficiencies and levels of total phenolics and activity of phenoloxidase and peroxidase in different groups. The whole experiments were repeated twice (McDonald, 2008).

\section{RESULTS \& DISCUSSION}

3.1. Confirming successful preparation of CsNPs by the Ionic Gelation Method

FTIR analysis showed that chitosan has two main characteristic peaks at $3433 \mathrm{~cm}^{-1}$ and $1644 \mathrm{~cm}^{-1}$ which correspond to stretching vibrations of the primary amine group $\left(-\mathrm{NH}_{2}\right)$ and the amide group (- $\left.\mathrm{CONH}_{2}\right)$, respectively; as shown in figure (1). On the other hand, FTIR analysis of chitosan nanoparticles showed that both peaks were shifted to $3428 \mathrm{~cm}^{-1}$ and $1580 \mathrm{~cm}^{-1}$, respectively; as shown in figure (2). 


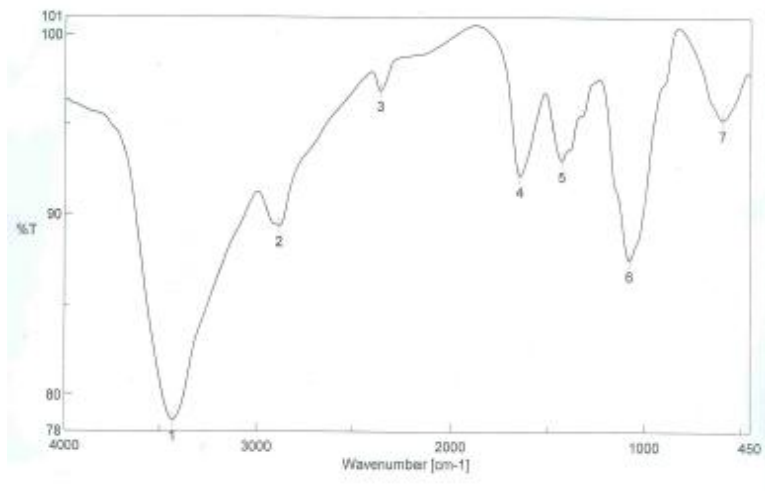

Fig.1: FTIR spectrum of chitosan

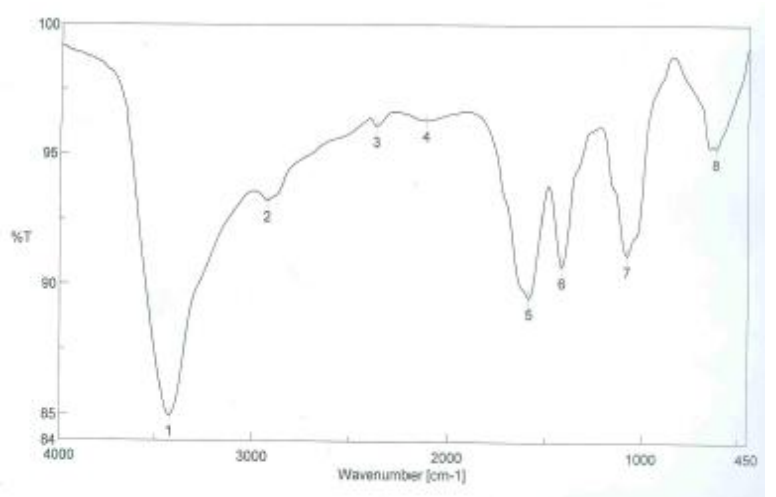

Fig.2: FTIR spectrum of chitosan nanoparticles

This reduction in stretching frequency indicates successful interaction of the polyphosphoric groups of sodium tripolyphosphate with the ammonium groups of chitosan and the more hydrogen bonding in chitosan-TPP complex.

Also, it is clear that the amine peak in chitosan nanoparticles (at $\mathbf{3 4 2 8} \mathrm{cm}^{-1}$ ) is sharper than the amine peak in chitosan (at $3433 \mathrm{~cm}^{-1}$ ), which indicates that the hydrogen bonding in chitosan nanoparticles was enhanced. (A. Anitha et al., 2009; Xu, Yongmei and Du, Yumin, 2003). Hence, chitosan nanoparticles were successfully synthesized by the ionic gelation method.

\subsection{Characterization of CsNPs}

Size distribution by number using dynamic light scattering revealed that the synthesized CsNPs have an average particle size about $\mathbf{5 0} \mathrm{nm}$ as shown in figure (3). In addition, transmission electron microscopy showed spherical shape of the synthesized CsNPs, as shown in figure (4).

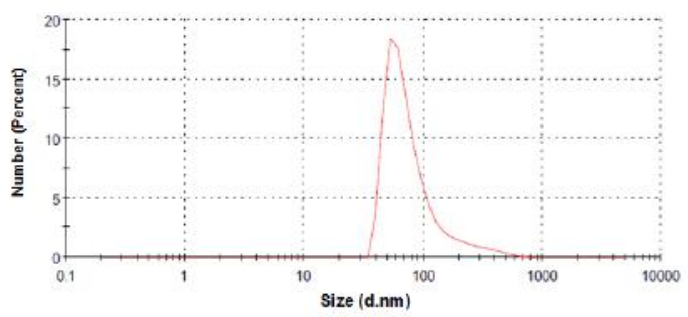

Fig.3: Particle size distribution of the synthesized CsNPs

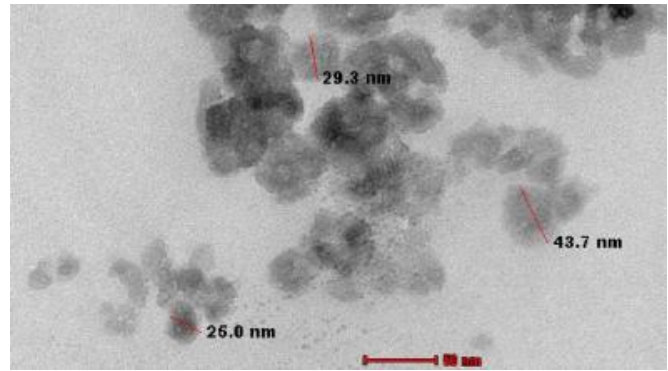

Fig.4: Transmission electron micrograph of the synthesized CsNPs, showing its spherical shape.

\subsection{Confirming successful preparation of CuNPs}

The Uv-vis spectroscopy of copper nanoparticles showed their characteristic resonance band at $\mathbf{5 7 2} \mathbf{~ n m}$, as shown in figure (5).

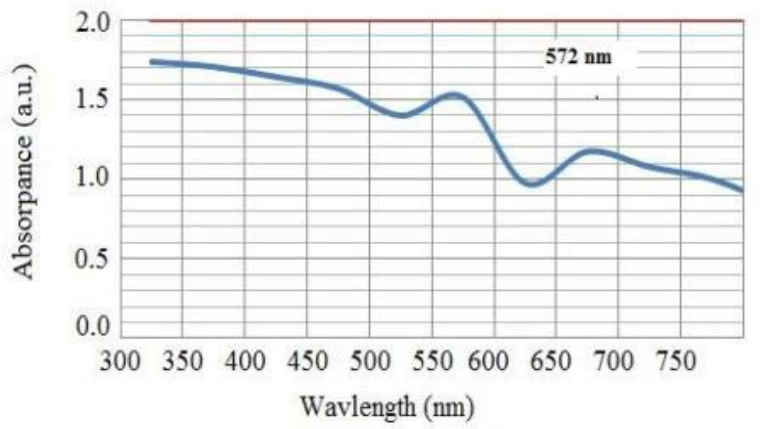

Fig.5: Uv-Vis spectrum of CuNPs showing its characteristic resonance peak at $572 \mathrm{~nm}$.

Also, X-ray diffraction pattern as shown in figure (6) revealed the main characteristic diffraction peaks at 2 theta $=43.36,50.47$ and 74.13 degrees, which correspond to the (1 11 1), ( $\left.\begin{array}{l}200 \\ 0\end{array}\right)$, and ( $\left.\begin{array}{lll}2 & 2 & 0\end{array}\right)$ crystal faces of copper (X. Zhu et al., 2012). It is also noteworthy that there are other peaks at 2 theta $=\mathbf{3 6 . 4 5}$ and $\mathbf{6 1 . 3 9}$ degrees, that are characteristic for $\mathrm{Cu}_{2} \mathrm{O}$ (M. S. M. Suan et al., 2011) which indicates the formation of $\mathrm{a}_{2} \mathrm{O}$ shell covering the $\mathrm{Cu}$ core due to surface oxidation of CuNPs. 


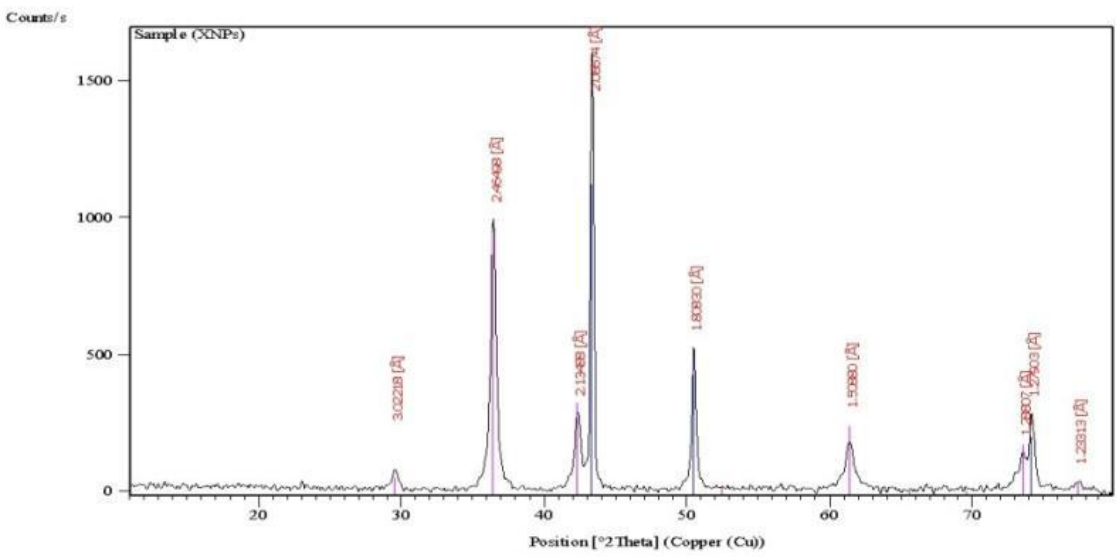

Fig.6: X-Ray Diffractogram of the synthesized CuNPs

\subsection{Characterization of CuNPs}

Transmission electron microscopy showed that the synthesized CuNPs have spherical shape, as shown in figure (7). Moreover, dynamic light scattering revealed that the average size of CuNPs was about $\mathbf{1 0 0} \mathrm{nm}$, as shown in figure (8).

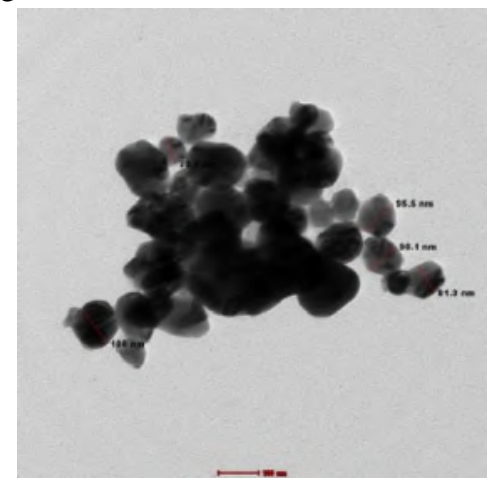

Fig.7: Transmission electron micrograph of copper nanoparticles, showing its spherical shape.

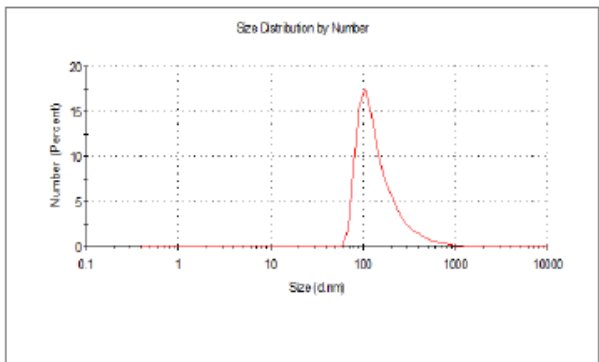

Fig.8: Size distribution by number of the synthesized

CuNPs.

Table.2: Positive immunomodulatory effect of CuCs on date palm seedlings

\begin{tabular}{|l|l|l|l|l|l|}
\hline \multirow{2}{*}{ Parameter } & \multicolumn{5}{|c|}{ Concentration of CuCs (g/l) } \\
\cline { 2 - 6 } & $\begin{array}{l}\mathbf{0 . 0 0} \\
\text { Control }\end{array}$ & $\mathbf{0 . 5 0}$ & $\mathbf{1 . 0 0}$ & $\mathbf{1 . 5 0}$ & $\mathbf{2 . 0 0}$ \\
\hline Total phenols $(\boldsymbol{m g} / \mathbf{g} \boldsymbol{d} \boldsymbol{w})$ & 2.70 & 2.92 & 3.29 & 3.62 & 4.05 \\
\hline $\begin{array}{l}\text { Phenoloxidase } \\
(\text { OD units } \mathbf{1 0} / \mathbf{m i n} / \mathbf{g d w})\end{array}$ & 31.4 & 33.83 & 40.22 & 47.10 & 62.73 \\
\hline $\begin{array}{l}\text { Peroxidase } \\
\left(\Delta \mathbf{O D}^{\mathbf{3}} \mathbf{1 0} / \mathbf{m i n} / \mathbf{g d w}\right)\end{array}$ & 40.33 & 66.03 & 96.52 & 109.85 & 121.53 \\
\hline
\end{tabular}


3.7. Evaluation of In vivo Efficiency of CopperChitosan Nanocomposition in Treating Fusarium Wilted-Date Palm Seedlings In Comparison With Rizolex $^{\text {tm }}$

Table (3) shows the summary of disease severity (DS) of the inoculated seedlings, which were treated with different concentrations of $\mathrm{CuCs}$ in comparison with those treated with water (-ve control) and with Rizolex ${ }^{\mathrm{TM}}$ (+ve control).

Statistical analysis showed that the disease severity of the inoculated date palm seedlings treated with different concentrations of the nanocomposition was significantly lower than that of the inoculated date palm seedlings which were treated with Rizolex $^{\text {TM }}$ (+ve Control) and water (-ve Control), except the concentration of $0.50 \mathrm{~g} / \mathrm{l}$.

Table.3: DS of the inoculated date palm seedlings treated with nanocomposition, Rizolex ${ }^{T M}$ and water.

\begin{tabular}{|l|l|l|l|l|l|l|}
\hline & \multicolumn{3}{|l|}{ Composition concentrations (g/l) } & $\begin{array}{l}\text { Rizolex } \\
\text { +ve Control }\end{array}$ & $\begin{array}{l}\text { Water } \\
\text {-ve Control }\end{array}$ \\
\cline { 2 - 5 } & $\mathbf{0 . 5 0}$ & $\mathbf{1 . 0 0}$ & $\mathbf{1 . 5 0}$ & $\mathbf{2 . 0 0}$ & & \\
\hline DS & 70.67 & 58.33 & 46.67 & 34.33 & 69.33 & 84.33 \\
\hline
\end{tabular}

\section{CONCLUSION}

To this point, it was clear that copper-chitosan nanocomposition may provide a competitive candidate for treating vascular wilt disease in date palm, which clearly manifested significantly higher efficiency than the commercially available fungicide at lower concentrations. This can be interpreted by virtue of two main axes:

Firstly, the positive immunomodulatory effect of copperchitosan nanocomposition on date palm seedlings as shown from its ability to increase peroxidase, phenoloxidase and total phenols levels, which were considered main constitutes of the plant innate immune response against the invading fungal pathogen;

Secondly, the antifungal effect of the nanocomposition on the fungal pathogen itself.

Hence, this copper - chitosan nanopcomposition with its dual complementary functionality may provide a potential approach to beleaguer the fusarium wilt disease in date palm via enhancing the plant immune responses at the same time of inhibiting the fungal growth. Thus, this nanocomposition can be used in developing new nanofungicides to control such pathogens. But, further research should be undertaken in order to investigate its potential toxicity on plant, human and environment; Thus, determining the optimal concentration that can be used in field without considerable toxicity consequences.

\section{REFERENCES}

[1] D. Karthik and K. Geetha, (2013). Synthesis of copper precursor, copper and its oxide nanoparticles by green chemical reduction method and its antimicrobial activity, Journal of Applied Pharmaceutical Science, vol. 3, no. 5, pp. 16-21.

[2] M. Muthukrishnan, (2015). Green synthesis of copper-chitosan nanoparticles and study of its antibacterial activity, Journal of Nanomedicine \& Nanotechnology, vol. 6, no. 1.
[3] Agrios, G.N. (2005). Plant Pathology, Elsevier Academic Press, San Diego, California, USA.

[4] Al-Shahib W. and R. J. Marshall. (2003). the fruit of the date palm: its possible use as the best food for the future. International Journal of Food Sciences and Nutrition, 54 (4): 247-259.

[5] Anitha, A., Rani, V. D., Krishna, R., Sreeja, V., Selvamurugan, N., Nair, S. V., ... \& Jayakumar, R. (2009). Synthesis, characterization, cytotoxicity and antibacterial studies of chitosan, Ocarboxymethyl and $\mathrm{N}$, O-carboxymethyl chitosan nanoparticles. Carbohydrate Polymers, 78(4), 672677.

[6] Campbell, C.L. and Madden, L.V. (1990). Introduction to Plant Disease Epidemiology, JohnWiley \& Sons, New York, NY, USA.

[7] Chandra S.; Chakraborty, N.; Dasgupta, A.; Sarkar, J. Panda, K. and Acharya, K. (2015). Chitosan nanoparticles: A positive modulator of innate immune responses in plants. Sci. Rep. 5, 15195; doi: 10.1038/srep15195 .

[8] Chester, K. S.; Horsfall, J. G. and Diamond, A. E. (1959). Plant Pathology: An AdvancesTraits, 1, Academic Press, New York, NY, pp. 199-242.

[9] Eva Petrikkou, Juan L. RodríguezTudela, Manuel Cuenca-Estrella, Alicia Gómez, Ana Molleja, and Emilia Mellado. (2001). Inoculum Standardization for Antifungal Susceptibility Testing of Filamentous Fungi Pathogenic for Humans. J. Clin. Microbiol. vol. 39 no. 4 1345-1347.

[10] F.A.O (2014). Dates Statistics 2014. Food and Agriculture Organization of the United Nations.

[11] Flood J. (2006). A review of Fusarium wilt of oil palm caused by Fusarium oxysporum f. sp. Elaeidis. Phytopathology, 96:660-662. 
[12] H. M. Yadav, S. V. Otari, V. B. Koli (2014). Preparation and characterization of copper-doped anatase $\mathrm{TiO}_{2}$ nanoparticles with visible light photocatalytic antibacterial activity, Journal of Photochemistry and Photobiology A: Chemistry, vol. 280, pp. 32-38.

[13] H. Tian, X. L. Zhang, J. Scott, C. Ng, and R. Amal, (2014). $\mathrm{TiO}_{2-}$ supported copper nanoparticles prepared via ion exchange for photocatalytic hydrogen production. Journal of Materials Chemistry A, vol. 2, no. 18, pp. 6432-6438.

[14] Hajipour, M. J.; Fromm, K. M. and Akbar Ashkarran A. (2012). Antibacterial properties of nanoparticles. Trends in Biotechnology. 30: 499511.

[15] Harish Prashanth KV and tharanathan RN (2007). Chitin/chitosan: modification and their unlimited application potential - an overview. Trends Food Sci. Techno. 18: 117-131.

[16] Hide G. A., P. J. Read and S. M. Hall (1992). Resistance to thiabendazole in Fusarium species isolated from potato tubers affected by dry rot. Plant Pathology, 41, 745-748.

[17] Ishaaya, I. (1971). Observations on the phenoloxidase system in the armored scales Aonidiella aurantii and Chrysomphalus aonidum. Comparative Biochemistry and Physiology Part B: Comparative Biochemistry, 39(4), 935-943.

[18] Kâhkônen , M.P.; Hopia, A.I.; Vuorela, H. J.; Rauha, J. P.; Pihlaja, K.; Kujala, T. S. and Heinonen, M. (1999). Antioxidant activity of plant extracts containing phenolic compounds. J.Agric,food chem., 47 :3954-3962.

[19] Ling Yien Ing, NoraziahMohamad Zin, Atif Sarwar, and Haliza Katas (2012). Antifungal Activity of Chitosan Nanoparticles and Correlation with Their Physical Properties. International Journal of Biomaterials, doi:10.1155/2012/632698

[20] M. S. M. Suan, M. R. Johan, N. L. Hawari, and H. A. Ching, (2011). Annealing effects on the properties of copper oxide thin films prepared by chemical deposition. International Journal of Electrochemical Science, vol. 6, no. 12, pp. 60946104.

[21] M. Sahu and P. Biswas. (2011). Single-step processing of copper-doped titania nanomaterials in a flame aerosol reactor. Nanoscale Research Letters, vol. 6, no. 1, article 441, pp. 1-14.

[22] McDonald J.H. (2008). Handbook of Biological Statistics Sparky House Publishing, Baltimore.

[23] Mustafa B. and Ilkay S. (2010). Controlled synthesis of copper nano/microstructures using ascorbic acid in aqueous CTAB solution. Powder Technology, 198, 279-284.
[24] Patel N., P. Desai, N. Patel, A. Jha and H. K. Gautam (2014). Agronanotechnology for plant fungal disease management: a review. Int J Curr Microbiol App Sci, 3:71-84.

[25] Pelgrift, R. Y. and Friedman, A. J. (2013). Nanotechnology as a therapeutic tool to combat microbial resistance. Advanced Drug Delivery Reviews. 65: 1803-1815.

[26] Prachi. K; Sonal, B.; Swapnil, G.; Aniket, G.; Amedea, B.; Seabrab, O.; Rubilarc, D.; Nelson.; Durane, F. and Mahendra R. (2013). In vitro antifungal efficacy of copper nanoparticles against selected crop pathogenic fungi. Materials Letters, 115, 13-17.

[27] Qi, L., Xu, Z., Jiang, X., Hu, C. and Zou, X. (2004). Preparation and antibacterial activity of chitosan nanoparticles. Carbohydr. Res. 339, 26932700.

[28] R. Betancourt-Galindo, P. Y. Reyes-Rodriguez, B. A. Puente Urbina et al., (2014). Synthesis of copper nanoparticles by thermal decomposition and their antimicrobial properties. Journal of Nanomaterials, vol. 2014, Article ID 980545, 5 pages.

[29] Riker, A.J. and Riker, R.S. (1936). Introduction to research on plant diseases. John's Swift Co., St. Louis, Chicago, New York and Indianapolis, 117 p.

[30] S. Magdassi, M. Grouchko, and A. Kamyshny, (2010). Copper nanoparticles for printed electronics: routes towards achieving oxidation stability. Materials, vol. 3, no. 9, pp. 4626-4638.

[31] S. T. H. Sherazi, R. A. Soomro, S. Uddin, and N. Memon, (2013). Synthesis and characterizations of highly efficient copper nanoparticles and their use in ultra fast catalytic degradation of organic dyes. Advanced Materials Research, vol. 829, pp. 93-99.

[32] Safiuddin Ansari1; Sheila Shahab; Mohd. Mazid and Dania Ahmed. (2012). Comparative study of Fusarium oxysporum f sp. lycopersici and Meloidogyne incognita race-2 on plant growth parameters of tomato. Agricultural Sciences 3: 844847.

[33] Singleton, V. L. and Rossi, J. A. (I965). Colorimetry of total phenolics with phosphomolybdic-phosphotungstic acid reagents. Am.J.Enol.Vitic., 16:144-158.

[34] Vetter, J. L., Steinberg, M. P., \& Nelson, A. I. (1958). Enzyme assay, quantitative determination of peroxidase in sweet corn. Journal of Agricultural and Food Chemistry, 6(1), 39-41.

[35] Wheeler, B.E.J. (1969). An Introduction to Plant Disease, John Wiley and Sons Limited,London. pp 301. 
[36] X. Zhu, B. Wang, F. Shi, and J. Nie, (2012).

Direct, rapid, facile photochemical method for preparing copper nanoparticles and copper patterns. Langmuir, vol. 28, no. 40, pp. 14461-14469.

[37] Xu, Yongmei and Du, Yumin (2003). Effect of molecular structure of chitosan on protein delivery properties of chitosan nanoparticles. International journal of pharmaceutics, Volume 250, Issue 1, 2 January 2003, Pages 215-226. 